\title{
Langzeiteffekt der stationären Rehabilitation bei Kindern und Jugendlichen mit mittelschwerem und schwerem Asthma bronchiale
}

\author{
C.-P. Bauer ${ }^{1}$ \\ F. Petermann² \\ D. Kiosz ${ }^{3}$ \\ R. Stachow ${ }^{4}$
}

\author{
Long-term Effect of Indoor Rehabilitation on Children and Young People \\ with Moderate and Severe Asthma
}

\section{Zusammenfassung}

In einer Multicenter-Studie wurde prospektiv der Effekt der stationären Rehabilitation bei Asthma bronchiale im Kindes- und Jugendalter im Vergleich zu einer ambulant betreuten Gruppe evaluiert.

In die Studie wurden 195 Kinder und Jugendliche mit Asthma bronchiale (Schweregrad 3 und 4) im Alter von 8 bis 16 Jahren aufgenommen. Die Reha-Gruppe (RG) umfasste 149 Patienten, die ambulante Vergleichsgruppe (VG) 46 Patienten. Erfasst wurde zu Beginn der Studie (bei der Reha-Gruppe ca. 2 Wochen vor Beginn der Reha) und 12 Monate später die Lungenfunktion $\left(\mathrm{FEV}_{1}\right.$, Peakflow und MEF50), asthmabedingte Schulfehlzeiten, Teilnahme am Schulsport, Asthma-Management und Lebensqualität.

12 Monate nach der stationären Reha zeigte die Reha-Gruppe im Vergleich zur ambulanten Gruppe signifikant bessere MEF50-Werte $(p=0,007)$. Parallel dazu zeigte die Reha-Gruppe im Asthma-Management (Wissen, Verhalten, Selbstwirksamkeit) ebenfalls signifikant bessere Werte $(p<0,01)$. Beim Schulsport gaben beide Gruppen mit 3,5 Wochenstunden bereits zu Beginn der Studie eine ausreichende Beteiligung an, die nicht weiter zu steigern war. Bei den asthmabedingten Schulfehltagen und der Lebensqualität zeigte die Reha-Gruppe zu Beginn der Studie schlechtere Ausgangswerte, die sich 12 Monate nach der

\section{Abstract}

In a multi-centre trial the effect of indoor rehabilitation in case of bronchial asthma in childhood and adolescence was evaluated on a prospective basis in comparison to an ambulant group of patients.

195 children and adolescents aged between 8 and 16 suffering from bronchial asthma (grading of severity 3 and 4) were included in the trial. The rehabilitation group (RG) included 149 patients, whereas the out-patient reference group consisted of 46 patients. At the beginning of the trial (in the rehabilitation group about 14 days before rehabilitation started) and 12 months afterwards, the pulmonary function ( $\mathrm{FEV}_{1}$, peak flow and MEF50), asthma-related school day off, participation in school sport, asthma management and quality of life were recorded.

12 months after the indoor rehabilitation, the rehabilitation group had significantly better MEF50-values $(P=0.007)$ than the ambulant group. The rehabilitation group also had significantly better values $(\mathrm{P}<0.01)$ with regard to the asthma management. Already at the beginning of the study both groups indicated a satisfactory participation in school sport with 3.5 hours a week which could not be increased further. The baseline values for asthma-related school day off and quality of life of the rehabilitation group were worse than those of the ambulant group. 12 months after rehabilitation, however, the values were signifi-

Institutsangaben

${ }^{1}$ Kinderklinik und Poliklinik der TU München, Bereich Rehabilitation und

Fachklinik Gaißach der LVA Oberbayern (Prof Dr. C.-P. Bauer)

${ }^{2}$ Zentrum für Rehabilitationsforschung der Universität Bremen (Prof. Dr. F. Petermann)

${ }^{3}$ Kinderfachklinik Satteldüne der LVA Schleswig-Holstein (Prof. Dr. D. Kiosz)

${ }^{4}$ Fachklinik Sylt der LVA Hamburg (Dr. D. Stachow)

Danksagung

Diese Studie wurde gefördert vom Verband der Deutschen Rentenversicherungsträger (VDR), der LVA Oberbayern, der LVA der Freien und Hansestadt Hamburg, der LVA Schleswig-Holstein, der Schleswig-Holsteinischen Vereinigung zur Bekämpfung der Tuberkulose und Lungenkrankheiten e.V. und dem Niedersächsischen Verein zur Bekämpfung der Tuberkulose-Lungen- und Bronchialerkrankungen e.V. Wir danken Herrn Dr. van Egmont-Fröhlich für die Mitwirkung bei der Erstellung des Studiendesigns.

Ebenfalls danken wir Frau PD Dr. P. Warschburger, Frau C. ten Thoren und Frau S. Busch für die Unterstützung bei der Auswertung der Daten.

Korrespondenzadresse

Univ.-Prof. Dr. C.-P. Bauer · Fachklinik der LVA Oberbayern · 83674 Gaißach 
Reha signifikant verbesserten $(\mathrm{p}<0,01)$ und sich dem Niveau der ambulanten Gruppe anglichen.

Durch eine stationäre Rehabilitation konnte somit eine signifikante Verbesserung im Bereich des MEF50, des Asthma-Managements, bei den Schulfehltagen und der Lebensqualität erreicht werden. cantly better $(\mathrm{P}<0.01)$ compared to baseline and almost similar to the levels of the ambulant group.

Significantly improvements with regard to MEF50, asthma management, school day off and quality of life could thus be achieved by indoor rehabilitation.

\section{Einleitung}

Das Asthma bronchiale stellt eine der häufigsten chronischen Erkrankungen im Kindesalter dar. Nach den Ergebnissen der ISAAC-Studie, einer internationalen Studie zur Prävalenz von Asthma und Allergien im Kindesalter, - besteht in Deutschland im Alter von 13 bis 14 Jahren eine 12-Monatsprävalenz von $14 \%$ für Asthma bronchiale [8].

Die Behandlung der Kinder mit Asthma bronchiale erfolgt in Deutschland primär beim Kinderarzt bzw. Hausarzt. Zusätzlich werden Kinder mit Asthma bronchiale vor allem bei schwereren Verläufen in Schwerpunktpraxen bzw. Spezialambulanzen betreut; bei akuter Verschlechterung erfolgt eine stationäre Therapie in der Klinik. Ergänzend dazu bestehen ambulante Angebote wie Asthmaschulung und Asthmasport, die allerdings bis heute nicht flächendeckend sind.

Falls mit dieser Versorgungskette ein zufriedenstellendes Behandlungsergebnis nicht zu erzielen ist, besteht die Möglichkeit einer stationären Rehabilitationsbehandlung. Die ambulante Behandlung sowie die akut stationäre Therapie ist eine Aufgabe der Krankenversicherung. Für die stationäre Rehabilitation dagegen ist primär der Rentenversicherer (LVA, BfA) zuständig [21].

Die Rehabilitation von Kindern und Jugendlichen wird als Aufgabe der Rentenversicherung gesehen, um u.a. chronisch kranken Kindern und Jugendlichen gute - möglichst mit Gesunden vergleichbare - Berufschancen zu ermöglichen.

Neben der Rentenversicherung können auch die Krankenkassen die Kosten für eine Rehabilitation übernehmen, sie sind jedoch nicht dazu verpflichtet.

Bei der stationären Rehabilitation steht die interdisziplinäre Betreuung durch Arzt, Schwester, Psychologe, Pädagoge und Physiotherapeut im Vordergrund. Bei Bedarf werden auch andere Berufsgruppen eingebunden.

Therapeutisch stehen neben der Optimierung der medikamentösen Behandlung vor allem die Anleitung zu einem besseren Umgang mit der chronischen Erkrankung im Vordergrund. Dazu stellen Patientenschulungen sowie Physio- und Sporttherapie unter Ausnutzung klimatischer Faktoren weitere Schwerpunkte dar. Hilfen zur Krankheitsbewältigung beziehen auch eine Berufsberatung bei Jugendlichen des entsprechenden Alters ein.

Im Rahmen einer Multicenter-Studie wurde deutschlandweit die Kurz- und Langzeit-Effektivität stationärer Rehabilitationsmaßnahmen bei Asthma bronchiale im Kindes- und Jugendalter anhand medizinischer und psychosozialer Parameter evaluiert.
Die Kurzzeiteffektivität der Rehabilitation, die durch Untersuchungen bei Aufnahme und bei Entlassung aus der Rehaklinik erfasst wurde und signifikante Verbesserungen in Bezug auf körperliche Leistungsfähigkeit, Lungenfunktion und Asthma-Management ergab, wurde bereits veröffentlicht [3].

Um darüber hinaus den Langzeiteffekt zu erfassen, wurde mittels Hausbesuch 2 Wochen vor und 12 Monate nach der Rehabilitation eine Lungenfunktionsdiagnostik durchgeführt und mittels standardisierter Fragebogen asthmabedingte Schulfehlzeiten, Teilnahme am Schulsport, Asthma-Management und Lebensqualität erfasst. Die dabei erhobenen Daten wurden den Ergebnissen einer ambulanten Vergleichsgruppe von Asthmatikern gleichen Schweregrades gegenübergestellt, die analog zur Rehagruppe untersucht wurde.

\section{Patienten und Methodilk}

\section{Patienten}

Von insgesamt 318 erfassten Kindern und Jugendlichen mit Asthma bronchiale im Alter von 8 bis 16 Jahren konnten zwischen April 1997 und April 1998195 Kinder und Jugendliche mit einem Asthma bronchiale der Stufe 3 und 4 in die Studie eingeschlossen werden. Diese teilten sich in eine Gruppe, die eine stationäre Rehabilitation erhielten (Rehagruppe $n=149$ ) mit einem durchschnittlichen Alter von 12,1 Jahren und eine ambulant betreute Gruppe (Vergleichsgruppe $n=46$ ) mit einem Altersdurchschnitt von 11,3 Jahren.

Die Erkrankungsdauer betrug in der Rehagruppe 7,39 Jahre, in der Vergleichsgruppe 7,46 Jahre.

In der Rehagruppe entfielen auf den Schweregrad 3 insgesamt 98 Patienten und auf den Schweregrad 4 insgesamt 51 Patienten. In der Vergleichsgruppe hatten 32 Patienten den Schweregrad 3 und 14 Patienten den Schweregrad 4.

56\% der Patienten der Rehagruppe und 72\% der Vergleichsgruppe waren männlich.

Die Schweregradeinteilung erfolgte nach den Richtlinien der Gesellschaft für Pädiatrische Pneumologie [4].

Ausschlusskriterien waren mangelhafte Kenntnisse der deutschen Sprache, Sonderschulbesuch der Patienten sowie Hörund Sprachschwierigkeiten und schwerwiegende andere Erkrankungen.

Eine Randomisierung zwischen Rehagruppe und ambulanter Vergleichsgruppe war nicht möglich, da Patienten mit einem be- 
willigten Rehabescheid einen Rechtsanspruch auf eine möglichst zeitnahe stationäre Rehabilitation haben. Sie konnten somit einer ambulanten Kontrollgruppe nicht zugeordnet werden. Es wurde deshalb eine ambulante Vergleichsgruppe aus den Patienten gebildet, die nach Einschätzung des Hausarztes ein Rehaverfahren benötigten, dafür aber bisher keinen Antrag gestellt hatten. Der häufigste Grund für den bisher nicht gestellten Antrag war die Sorge vor Schulversäumnis, obwohl auch während der Rehamaßnahme ein Schulunterricht in der Klinik stattfindet.

Die Patienten der Rehagruppe hatten in Absprache mit ihrem Hausarzt unabhängig von der Studie bereits einen Antrag beim Rentenversicherungsträger gestellt. Nach Prüfung und Bewilligung wurde der Bescheid den jeweiligen Kliniken zugeleitet. Von den Kliniken wurden die Patienten vor Beginn der Rehabilitation zur Studie schriftlich eingeladen und wenn die Einverständniserklärung zur Teilnahme vorlag, wurden die Familien von speziell für die Studie geschulten Feldforschern zu Hause untersucht. Die gleiche Untersuchung fand 12 Monate später nochmals statt.

Die Patienten der ambulanten Vergleichsgruppe wurden von 116 Kinder- und Hausärzten in Nord- und Süddeutschland rekrutiert, die über die Studie im Vorfeld informiert waren und über entsprechende Studienunterlagen verfügten.

Patienten, die nach oben beschriebener Einschätzung infrage kamen, wurden vom Hausarzt zur Teilnahme an der ambulanten Vergleichsgruppe eingeladen. Nach Vorliegen des schriftlichen Einverständnisses wurden sie unmittelbar danach ebenfalls von den Feldforschern und nach weiteren 12 Monaten analog zu den Patienten der Rehagruppe im Rahmen eines Hausbesuches untersucht.

\section{Therapiekonzept}

Das Rehabilitationsprogramm wurde entsprechend dem Rahmenkonzept des Verbandes Deutscher Rentenversicherer (VDR) zur stationären Rehabilitation von Kindern und Jugendlichen bei der Indikation Asthma bronchiale durchgeführt $[2,20]$.

Die Rehabilitation erfolgte in der Fachklinik Gaißach der LVA Oberbayern, der Kinderfachklinik Satteldüne der LVA SchleswigHolstein und der Fachklinik Sylt der LVA Hamburg.

Die ambulante Vergleichsgruppe wurde vom Hausarzt weiterbetreut und erhielt neben der bereits bestehenden Therapie kein studienspezifisches Interventionsprogramm.

\section{Studienparameter}

Zur Objektivierung des klinischen Verlaufes erfassten die Feldforscher im Rahmen des Hausbesuches folgende Parameter: $\mathrm{FEV}_{1}$, FVC, Peakflow und MEF50. Die Daten wurden mit einem Lungenfunktionsgerät der Firma Jäger (Flowscreen) erhoben und als Normwerte dienten die Werte von Zapletal [27]. Es wurden mindestens drei und maximal acht Messungen vorgenommen. Kriterium für die Genauigkeit der Messungen war, dass die Differenz zwischen dem höchsten und zweithöchsten gemessenen FVC- und $\mathrm{FEV}_{1}$-Wert nicht mehr als $5 \%$ und bei dem PEFWert nicht mehr als $10 \%$ betrug $[1,19]$.
Die Messungen erfolgten jeweils zur selben Tageszeit und am Untersuchungstag hatte der Patient mindestens 12 Stunden zuvor keine atemwegserweiternden Medikamente eingenommen.

Mittels schriftlicher Interviews wurden die asthmabedingten Schulfehlzeiten, die Teilnahme am Schulsport, das Asthma-Management (Wissen, Verhalten, Selbstwirksamkeit) und die gesundheitsbezogene Lebensqualität erfasst.

Das asthmaspezifische Wissen und das Selbstwirksamkeitserleben konnte durch neuentwickelte Fragebogen erfasst werden. Im Bereich asthmaspezifisches Wissen (Sport, Dauermedikation, Notfall- und Auslöserwissen) erhielten die Kinder und Jugendlichen für jede richtige Antwort einen Punkt. Die Patienten konnten hier maximal 26 Punkte erreichen. Zur Erfassung des Selbstwirksamkeitserlebens schätzten sie anhand einer 5-stufigen Antwortskala ihre Fertigkeiten ein, das Asthma zu kontrollieren. Der Mittelwert wurde an der Anzahl der beantworteten Items relativiert und variierte zwischen 1 und 5.

Die Verhaltenskompetenzen (korrekte Anwendung eines Notfallpräparates, atemerleichternde Stellungen und Lippenbremse) mussten die Patienten den Feldforschern demonstrieren. Jeder korrekte Schritt in der Durchführung der einzelnen Verhaltensweisen wurde mit einem Punkt bewertet. Der Summenwert wurde dann wieder durch die Anzahl der Items geteilt, so dass sich der relativierte Mittelwert zwischen 0 und 1 bewegte.

Die gesundheitsbezogene Lebensqualität wurde mit einer deutschen Übersetzung des „Pediatric Asthma Quality of Life Questionnaire“ von Juniper erfasst [9]. Der Multidimensionalität der gesundheitsbezogenen Lebensqualität wurde dabei zunächst mit folgenden 3 Unterteilungen Rechnung getragen: Beeinträchtigung der Aktivitäten, Emotionen und Beeinträchtigung aufgrund der Symptomatik. Im Bereich Aktivitäten wurden individuelle Einschränkungen gemessen, wobei die Kinder und Jugendlichen 5 Tätigkeiten nennen konnten, in denen sie sich besonders durch ihr Asthma beeinträchtigt fühlten.

\section{Statistik}

Um die Vergleichbarkeit der Untersuchungsgruppen hinsichtlich soziodemografischer und ausgewählter krankheitsspezifischer Variablen zu prüfen, wurden $\mathrm{Chi}^{2}$ - bzw. t-Tests für unabhängige Stichproben berechnet. Die Verlaufsdaten wurden varianzanalytisch ausgewertet, wobei das Signifikanzniveau entsprechend den Konventionen in der psychologischen Forschung auf $5 \%$ festgelegt war. Da die meisten Parameter multidimensional vorliegen (z.B. Krankheitsmanagement und Lebensqualität), wurde eine multivariate Varianzanalyse (MANOVA) mit anschließenden univariaten Tests als Methode der Wahl eingesetzt, um den Alphafehler möglichst gering zu halten [10]. Einer Testung auf Unterschiede im Gesamtkonstrukt folgte dann die Prüfung, ob sich auch in den einzelnen Bereichen dieses Parameters Veränderungen über die Zeit, Gruppenunterschiede bzw. wechselseitige Abhängigkeiten zeigten. Weiterhin wurden sowohl für die einzelnen Messzeitpunkte als auch für die Gruppen Bonforoni korrigierte paarweise Vergleiche angeschlossen. 
Tab. 1 Soziodemograpische Parameter in der Rehabilitations- und Vergleichsgruppe bei T0

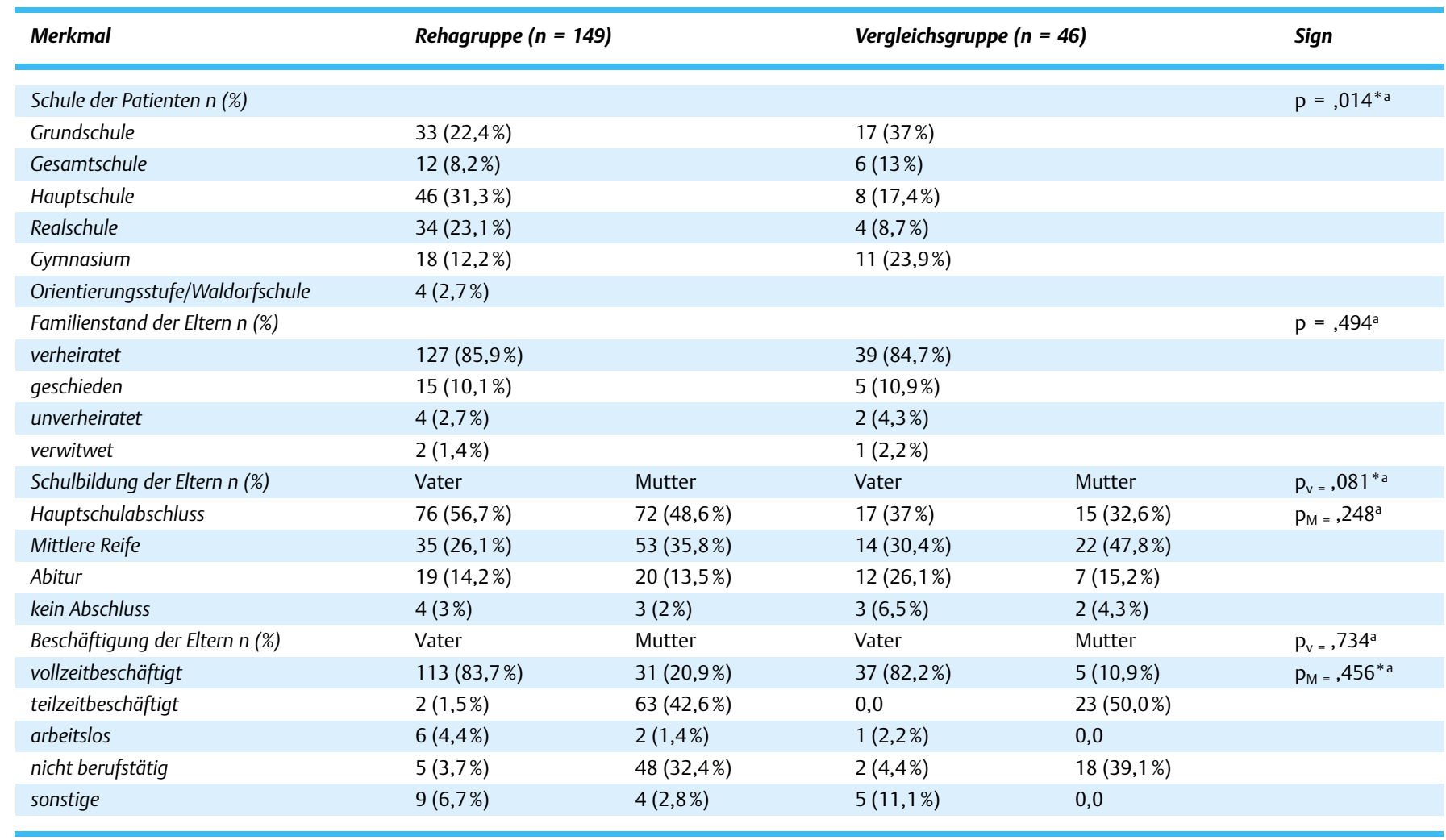

a Analyse mit $\chi$-Quadrat-Test

\section{Ergebnisse}

\section{Basisdaten}

Von den ursprünglich in die Rehagruppe eingeschlossenen 149 Patienten des Schweregrades 3 und 4 beendeten 140 Patienten die Studie.

In die Vergleichsgruppe waren 46 Patienten des Schweregrades 3 und 4 eingeschlossen und 42 beendeten die Studie. Es handelte sich dabei nicht um selektive Dropouts. 123 von 149 Patienten der Rehagruppe und 44 von 45 Patienten der Vergleichsgruppe erhielten bereits vor Beginn der Studie eine Dauermediaktion. Diese Medikation wurde an den Untersuchungstagen (Lungenfunktion) selbst abgesetzt.

Die Verteilung der soziodemographischen Parameter (Schultyp der Patienten, Familienstand, Schulbildung und Beschäftigung der Eltern) ist in Tab.1 dargestellt. Es zeigte sich lediglich, dass in der Rehagruppe prozentual mehr Hauptschüler und Realschüler, dagegen in der Vergleichsgruppe mehr Gymnasiasten vertreten waren $(p=0,14)$. Bezüglich Familienstand, Schulbildung und Beschäftigung der Eltern gab es keine Unterschiede zwischen den Gruppen.

\section{Lungenfunktionsdaten}

Die Bestimmung des $\mathrm{FEV}_{1}$ in der Rehagruppe ergab zu Beginn der Studie (T0) einen Mittelwert von 86,4\% vom Soll (SEM = 1,4) und in der Vergleichsgruppe von 84,8\% (SEM = 3,6). 12 Monate später lag der Mittelwert für die Rehagruppe bei 92,8\% (SEM = 1,4) und für die Vergleichsgruppe bei 87,3\% (SEM =2,5).

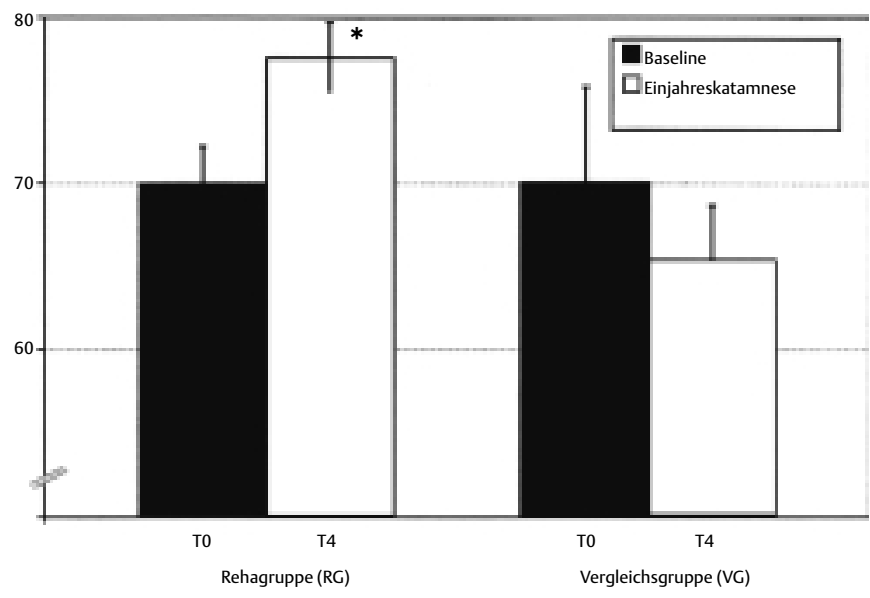

Abb. 1 MEF 50 in \% vom Soll der Reha und Vergleichsgruppe zu $T_{0}$ und $\mathrm{T}_{4}\left(\mathrm{~T}_{0}: \mathrm{RG}=70 \%, \mathrm{VG}=70,1 \% ; \mathrm{T}_{4}: \mathrm{RG}=77,6 \%, \mathrm{VG}=65,4 \%\right)^{*} \mathrm{p}<0,001$.

Statistisch ergab sich kein signifikanter Unterschied zwischen beiden Gruppen für diesen Parameter. Auch für die FVC und die Peakflow-Werte bestand weder zu Beginn der Studie noch 12 Monate später ein signifikanter Unterschied zwischen beiden Gruppen (Tab. 2).

Bei der Ermittlung des MEF50 ergab sich für die Rehagruppe zu Beginn der Studie ein Mittelwert von 70,0\% (SEM = 2,2) und für die Vergleichsgruppe ein Mittelwert von 70,1\% (SEM = 5,8).

12 Monate später stieg der MEF50-Wert in der Rehagruppe signifikant auf 77,6\% (SEM=2,2) an ( $\mathrm{p}<0,001)$. Der MEF50-Wert der Vergleichsgruppe nahm im Mittel auf 65,4\% (SEM = 3,3) ab. 
Tab. 2 Mittelwerte der Lungenfunktionsparameter FCV, FVC und PEF

\begin{tabular}{|c|c|c|c|c|c|}
\hline \multirow[t]{2}{*}{ Lungenfunktionsparameter } & \multirow[t]{2}{*}{ Gruppe } & \multicolumn{2}{|c|}{ Mittelwert } & \multicolumn{2}{|l|}{ SEM } \\
\hline & & TO & T4 & TO & T4 \\
\hline $\mathrm{FEV}_{1} \%$ Soll & Vergleichsgruppe & 84,8 & 87,4 & 3,6 & 2,5 \\
\hline \multirow[t]{2}{*}{ FVC \% Soll } & Rehagruppe & 86,8 & 93,4 & 1,2 & 1,3 \\
\hline & Vergleichsgruppe & 84,9 & 89,0 & 3,0 & 2,5 \\
\hline
\end{tabular}

T0: Studienbeginn; T4: nach 12 Monaten

Die MEF50-Werte der Rehagruppe unterschieden sich nach 12 Monaten signifikant von den MEF50-Werten der Vergleichsgruppe $(\mathrm{p}=0,007)$.

Es war somit in der Rehagruppe nach 12 Monaten zu signifikant besseren MEF50-Werten im Vergleich zur Vergleichsgruppe gekommen (Abb.1).

\section{Entwicklung der Schulfehltage}

Kinder und Jugendliche der Rehagruppe gaben bei Einschluss in die Studie für den Zeitraum 12 Monate vor Studienbeginn im Durchschnitt wesentlich mehr asthmabedingte Schulfehltage ( $M=11,9$, Range 0 - 70) an, als Patienten der ambulant behandelten Vergleichsgruppe ( $M=4,0$, Range 0-50). Über die Messzeitpunkte gemittelt ergab sich ein signifikanter Gruppenunterschied $\left(F=9,6 ; p<0,01 ; \eta^{2}=0,051\right)$. Für den Zeitraum 12 Monate nach Studieneinschluss konnten die stationär behandelten Kinder und Jugendlichen die asthmabedingten Schulfehltage reduzieren und dem Niveau der Vergleichsgruppe annähern. Diese Abnahme der Schulfehltage in der Rehagruppe war signifikant $\left(F=7,79 ; p<0,01 ; \eta^{2}=0,042\right)$ (Abb. 2).

\section{Entwicklung der Teilnahme am Schulsport}

Bei der Auswertung der Teilnahme am Schulsport gaben die Patienten der Rehagruppe vor Studienbeginn durchschnittlich 3,5 Stunden pro Woche an; die Vergleichsgruppe 3,8 Stunden. Nach 12 Monaten gaben sowohl Rehagruppe als auch Vergleichsgruppe durchschnittlich 3,5 Wochenstunden Teilnahme am Schulsport an. Somit nahmen die Patienten bereits zu Beginn der Stu-

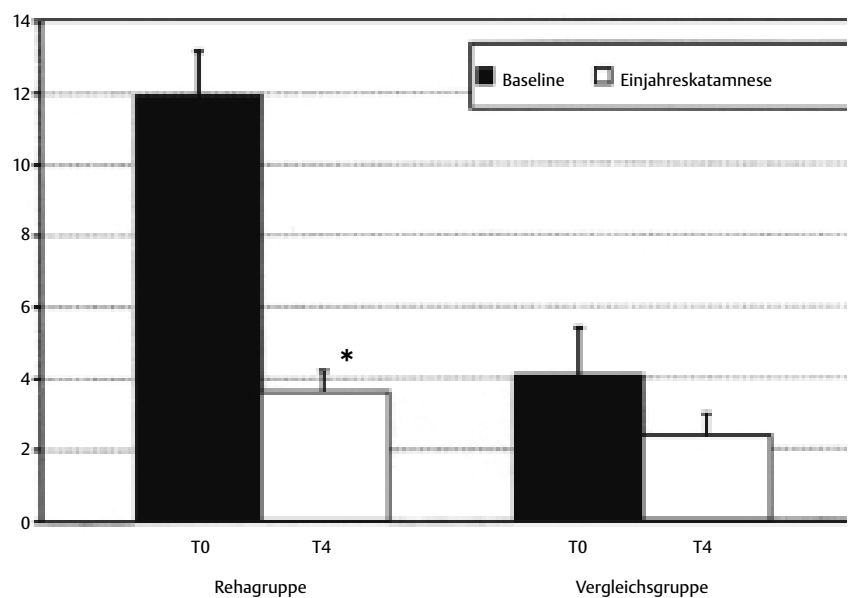

Abb. 2 Schulfehltage der Reha- und Vergleichsgruppe zu $T_{0}$ und $T_{4}$. * $p<0,01$. die in beiden Gruppen in hohem Maße am Schulsport teil und dies war durch die Rehabilitation nicht mehr zu steigern.

\section{Krankheitsmanagement}

In Bezug auf das Krankheitsmanagement (Wissen, Verhalten, Selbstwirksamkeit) zeigten die Patienten der Rehagruppe und Vergleichsgruppe in den Bereichen Wissen (Auslöser, Medikation, Ausübung von Sport etc.) und Verhalten (Lippenbremse, atemerleichternde Stellungen, Lungendetektiv und Medikamentenapplikation) bei Einschluss in die Studie nur geringe Kenntnisse. So erreichte die Rehagruppe bei T0 für den Bereich Wissen einen Mittelwert von 12,6 (SEM = 0,3) von 26 Punkten. Die Vergleichsgruppe hatte bei T0 einen Mittelwert von 12,2 (SEM =0,5).

In dem 12-monatigen Beobachtungszeitraum verbesserte sich die Rehagruppe signifikant $(\mathrm{p}<0,01)$ auf einen Mittelwert von $M=16,0$ (SEM =0,3). Die Ergebnisse des Wissenstests der Vergleichsgruppe dagegen zeigten nur eine geringfügige, nicht signifikante Änderung $(\mathrm{M}=12,9, \mathrm{SEM}=0,6)$. Der Unterschied zwischen beiden Gruppen nach 12 Monaten war signifikant $(p<0,01$, S. Abb. 3).

Für den Bereich Verhalten zeigte sich ein ähnlicher Verlauf. Bei Studienbeginn lag der Verhaltenswert für die Rehagruppe bei $M=0,4 \quad(S E M=0,02)$, für die Vergleichsgruppe bei $M=0,5$ ( $\mathrm{SEM}=0,03$ ). 12 Monate nach Studieneinschluss stieg in der Rehagruppe der Mittelwert für asthmaspezifisches Verhalten auf $\mathrm{M}=0,8(\mathrm{SEM}=0,01)$ an. In der Vergleichsgruppe lag der Wert 12 Monate nach Rehabeginn bei $\mathrm{M}=0,6(\mathrm{SEM}=0,03)$.

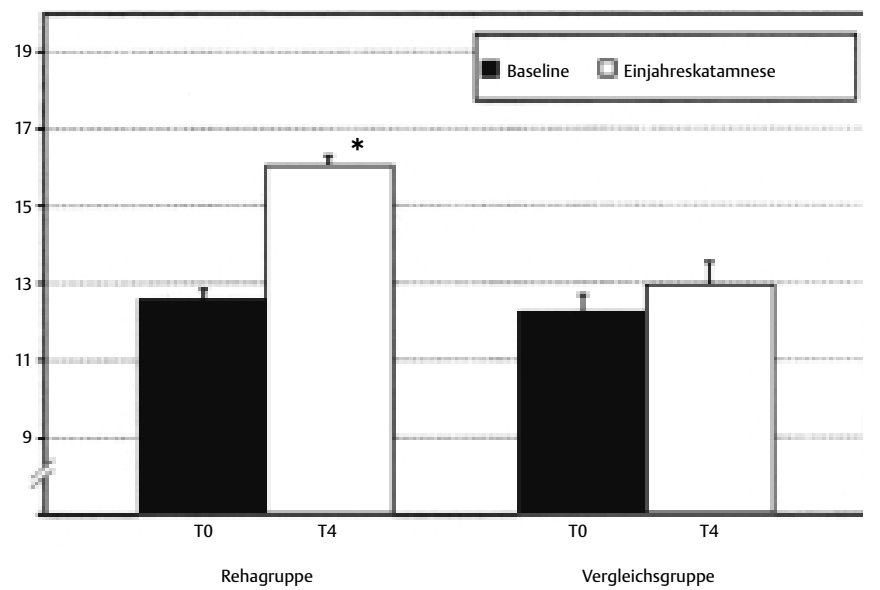

Abb. 3 Asthmaspezifisches Wissen der Reha- und Vergleichsgruppe $\mathrm{zu} \mathrm{T}_{0}$ und $\mathrm{T}_{4} \cdot{ }^{*} \mathrm{p}<0,01$. 


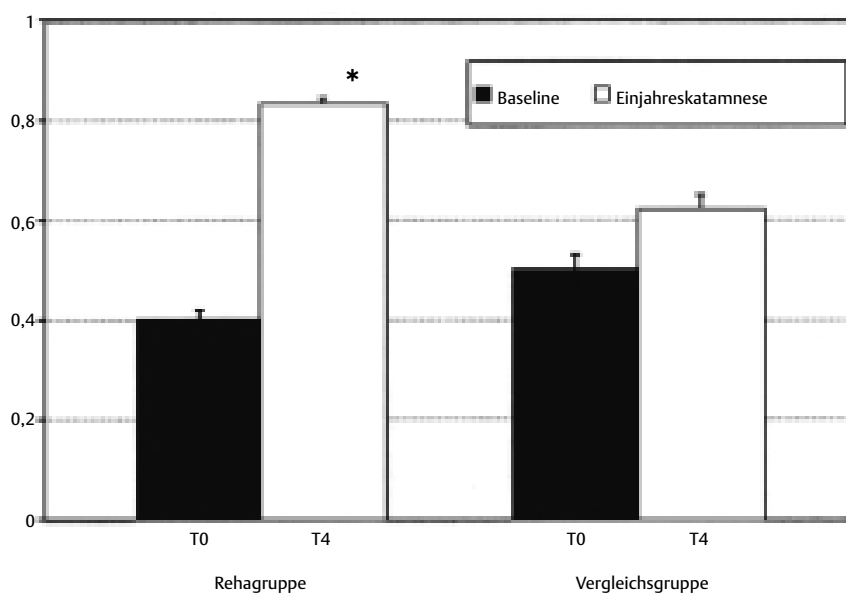

Abb. 4 Asthmaspezifische Verhaltensfertigkeiten der Reha- und Vergleichsgruppe zu $\mathrm{T}_{0}$ und $\mathrm{T}_{4}$. ${ }^{*} \mathrm{p}<0,01$.

Die Verbesserung im Bereich Verhalten war in der Rehagruppe signifikant $(p<0,01)$ und der Unterschied zwischen der Rehagruppe und der Vergleichsgruppe nach 12 Monaten war ebenfalls signifikant ( $p<0,01$, s. Abb. 4).

Bei der Erfassung der Selbstwirksamkeit schätzten die Kinder ihre Möglichkeiten, das Asthma kontrollieren zu können, bereits zu Studienbeginn hoch ein (Rehagruppe $M=3,0, S E M=0,5$; Vergleichsgruppe $M=3,7, \operatorname{SEM}=0,1$ ).

12 Monate nach Beginn der Studie zeigte die Rehagruppe einen Mittelwert von $M=4,0$, SEM $=0,05$, die ambulante Vergleichsgruppe dagegen einen Mittelwert von $\mathrm{M}=3,8, \mathrm{SEM}=0,09$.

Obwohl die Kinder ihre Möglichkeiten bereits selbst als gut eingeschätzt hatten, konnte die Rehagruppe im Vergleich zu den ambulant behandelten Patienten ihre Selbstwirksamkeit noch signifikant steigern $(p<0,01)$.

\section{Lebensqualität}

Schon zu Beginn der Untersuchung berichteten die Patienten beider Untersuchungsgruppen sowohl im Gesamtwert (Rehagruppe: $M=3,6, S E M=0,07$; Vergleichsgruppe: $M=4,0, S E M=0,1)$ als auch in den einzelnen Bereichen über eine relativ hohe Lebensqualität. Dennoch nahm bei den ambulant und den stationär behandelten Patienten die gesundheitsbezogene Lebensqualität über den Zeitraum eines Jahres noch zu (Rehagruppe: $M=4,4$, SEM =0,05; Vergleichsgruppe: $M=4,3, \operatorname{SEM}=0,1$ ). Der größere Zuwachs in der Rehabilitationsgruppe schlägt sich in der signifikanten Interaktion nieder $\left(F=16,2 ; p<0,01 ; \eta^{2}=0,104\right)$. Lag das Niveau der Rehabilitationsgruppe zum ersten Messzeitpunkt noch unter dem der Vergleichsgruppe, haben die stationär behandelten Patienten in der Einjahreskatamnese die Vergleichsgruppe erreicht. Betrachtet man die einzelnen Bereiche der krankheitsspezifischen Lebensqualität, so findet der größte Zuwachs über die Zeit in den Tätigkeiten statt, in denen die Patienten zu Beginn die größten Einschränkungen erlebten.

\section{Diskussion}

Die Rehabilitation von Kindern und Jugendlichen hat sich in den letzten 15 Jahren deutlich geändert. Stand der Klimaeffekt vor Jahren bei solchen Maßnahmen noch im Vordergrund, sind heute neben der Optimierung der medikamentösen Therapie der Sport, die Physiotherapie, die Patientenschulung und Hilfen zur Krankheitsbewältigung wesentliche Inhalte solcher Maßnahmen [20]. Unterstützend kann sich dabei sicher ein günstiges, z.B. allergenarmes, Klima auswirken. Der Therapieansatz der stationären Rehabilitation ist interdisziplinär, ambulant werden heute auch Teile der stationären Reha meist in Form von Schulungsprogrammen und speziellen Sportmöglichkeiten („Asthmasport“) angeboten. Die Effektivität solcher ambulanter Maßnahmen wurde bereits mehrfach untersucht und Zielparameter solcher Studien waren in der Regel Schulversäumnis und Verbesserung des sog. Asthmawissens. Diese Studien konnten mehrheitlich eine Reduktion der Schulversäumnisse und eine Vermehrung des „Asthmawissens“ nachweisen [6,7,14,15,24].

Solche ambulanten Schulungsangebote decken aber nur Teilbereiche der stationären Rehabilitation ab und können deshalb für schwerer Betroffene den Inhalt und das Ziel der stationären Rehabilitation nicht ersetzen.

Trotz verschiedenster ambulanter Angebote wird nicht nur in Deutschland, sondern auch international z.B. in USA, Frankreich, Belgien, Schweiz, Norwegen, Polen, u. a. eine stationäre Rehabilitation durchgeführt. Die medizinische Effektivität und auch der langfristige ökonomische Nutzen für das Gesundheitssystem konnte von Weinstein und Mitarbeitern aufgezeigt werden [25]. Hier kam es durch eine stationäre Rehabilitation bei Kindern mit schwerem Asthma in einer Beobachtung über 4 Jahre zu einer signifikanten Reduktion der Behandlungskosten der Patienten in diesem Zeitraum. Eine Übersicht über den positiven Effekt der stationären Rehabilitation bei chronisch obstruktiven Lungenerkrankungen im Erwachsenenalter gibt die Meta-Analyse von Lacasse [11]. Ebenfalls für das Erwachsenenalter zeigt Petro [17] die Effekte der pneumologischen Rehabilitation auf.

Da für die in Deutschland durchgeführten stationären Rehamaßnahmen im Kindes- und Jugendalter bisher keine mittel- und langfristigen Ergebnisdaten vorlagen, war es das Ziel dieser Studie zu prüfen, ob durch eine Rehamaßnahme bei Asthma bronchiale die Lungenfunktion, das Asthma-Management und psychosoziale Faktoren langfristig gebessert werden können. Um diese Frage zu beantworten, sollten neben dem Prä-Postvergleich der Rehagruppe die Ergebnisse auch mit Verlaufsdaten ambulant betreuter Patienten gleichen Krankheitsschweregrades verglichen werden.

Die Bildung einer „klassischen“ randomisierten Kontrollgruppe war aus versicherungsrechtlichen Gründen - wie vorher dargestellt - nicht möglich. Der zahlenmäßige Unterschied zwischen Reha- und Vergleichsgruppe ergab sich aus dem Verlauf der Studie. In 116 angesprochenen Praxen in Nord- und Süddeutschland konnten in dem vorgegebenen Rekrutierungszeitrahmen nur 46 Patienten mit Asthmaschweregrad 3 und 4 in die Vergleichsgruppe eingeschlossen werden, die aus Sicht des Hausarztes eine Rehabilitation benötigten, diese jedoch nicht in Anspruch nahmen. 
Ein besonderer Aspekt dieser Studie ist es, dass erstmalig im Bereich der Kinderrehabilitation die Daten außerhalb der Klinik vor und 12 Monate nach der Reha durch Hausbesuche erhoben wurden. Durch die Hausbesuche konnte sichergestellt werden, dass die Lungenfunktionsuntersuchung vor und nach der Reha standardisiert erfolgte und die Fragebogen zur Erfassung der psychosozialen Parameter von den Patienten selbst, unabhängig von den Eltern, ausgefüllt wurden. Bei der Auswertung der Lungenfunktionsparameter zeigte sich, dass sich die Gruppen im $\mathrm{FEV}_{1}$-Wert, Peakflow und FVC-Wert nicht unterscheiden und in beiden Gruppen im Normbereich lagen. Hier ist zu berücksichtigen, dass die überwiegende Mehrzahl der Patienten in beiden Gruppen bereits vor der Reha eine Dauermedikation des Asthma bronchiale erhielt, die allerdings am Untersuchungstag abgesetzt war.

Bei der Messung des MEF50 lagen beide Gruppen zu Beginn der Studie im Grenzbereich zu den pathologischen Werten [16]. Auffällig ist, dass die MEF50-Werte der Rehagruppe sich über 12 Monate in den Normbereich hinein stabilisierten. Die Werte der Vergleichsgruppe dagegen verschlechterten sich noch leicht und lagen nach 12 Monaten im beginnnenden pathologischen Bereich. Dieser Unterschied zwischen beiden Gruppen im Verlauf war statistisch signifikant.

Da Lungenfunktionsergebnisse zirkadianen Schwankungen unterworfen sind [26], wurde darauf geachtet, dass die Messungen zur gleichen Tageszeit durchgeführt wurden. Da ein Abstand von 12 Monaten zwischen beiden Messungen lag, war auch gewährleistet, dass die Untersuchung zur gleichen Jahreszeit war und die Messung selbst wurde jeweils in der Wohnung des Patienten durchgeführt.

Durch diese Daten wird aufgezeigt, dass der $\mathrm{FEV}_{1}$-Wert zur Einschätzung des Langzeitverlaufes eines Asthma bronchiale alleine nicht ausreichend ist. Als Messverfahren zur Überwachung eines Asthmatikers sollte deshalb mindestens immer eine Flussvolumenuntersuchung erfolgen.

$\mathrm{Zu}$ diskutieren ist, ob die Verbesserung der MEF50-Werte der Rehagruppe auf eine Verbesserung der Compliance und Inhalationstechnik, eine konsequentere und intensivere antientzündliche Therapie und Reduzierung der Allergen- und Schadstoffexposition (z. B. Nikotin) im häuslichen Milieu zurückzuführen ist.

Alle diese Punkte wurden therapeutisch durch das Rehaverfahren angegangen. Dass dabei das Asthma-Management der Rehagruppe im Vergleich zur Vergleichsgruppe über den Zeitraum von 12 Monaten besonders verbessert wurde, zeigen die Ergebnisse des Asthmaverhaltens. Bei Asthmawissen kam es auch zu einer signifikanten Verbesserung der Rehagruppe. Eine weitere Verbesserung der 12-Monatsergebnisse erscheint hier jedoch erforderlich und es ist eine gezielte ambulante Nachschulung in diesem Bereich zu diskutieren.

Überraschend war, dass im Bereich der Teilnahme am Schulsport in beiden Gruppen keine wesentliche Einschränkung nachweisbar war. So sind heute z.B. in Bayern 4 Stunden Schulsport die Regel, die von der Mehrzahl der Studienteilnehmer bereits vor Beginn der Studie fast erreicht wurde. Gegenüber früheren Untersuchungen $[12,13]$ ist hier eine Verbesserung zu verzeichnen, die möglicherweise auf eine insgesamt verbesserte Asthmabetreuung und eine intensive Öffentlichkeitsarbeit auf diesem Gebiet in den letzten Jahren zurückzuführen ist.

Deutliche asthmabedingte Fehlzeiten beim Schulbesuch hatten dagegen die Patienten der Rehagruppe im Vergleich zur ambulanten Gruppe. Die Rehagruppe erreichte hier über 12 Monate eine signifikante Reduzierung der Schulfehltage und Angleichung an die Vergleichsgruppe. Ebenfalls kam es zu einer signifikanten Verbesserung der Lebensqualität. Die vor der Rehabilitation bestehenden Unterschiede zwischen Reha- und Vergleichsgruppe waren nach 12 Monaten nicht mehr nachweisbar.

Nach diesen Ergebnissen ist davon auszugehen, dass die Patienten, die eine Rehabilitation in Anspruch nehmen, innerhalb der Asthmatiker vergleichbaren Schweregrades eine besondere Gruppe mit Einschränkungen im psychosozialen Bereich darstellen. Ihre Prognose ohne Rehabilitation ist deutlich ungünstiger zu stellen. Umso mehr ist zu betonen, dass bei ungünstigeren Ausgangsbedingungen (Schulfehltage, Lebensqualität) sich 12 Monate nach der Rehabilitation die Unterschiede ausgleichen und die Prognose aufgrund der Lungenfunktion für die Rehagruppe sogar günstiger ausfällt. Danach lässt sich hoffen, dass Patienten mit Asthma bronchiale und einer primär ungünstigeren medizinischen Prognose durch die Rehabilitation der Übergang vom Jugend- zum Erwachsenenalter problemloser gelingt.

\section{Literatur}

${ }^{1}$ American Thoracic Society. Standardisation of Spirometrie. American J of Resp Crit Care Med 1995; 152: 1107-1136

${ }^{2}$ Bauer CP, van Egmond-Fröhlich A, Eckl F, Fichtner O, Stein D. Kinderrehabilitation bei Asthma bronchiale. Kinderarzt 1996; 9: 1093 - 1100

${ }^{3}$ Bauer CP. Erste Ergebnisse der ESTAR-Studie. Südd. Kinderärzte-Kongress München, 2000

4 Berdel D, Reinhardt D, Hofmann D, Leupold W, Lindemann H. Therapieempfehlungen der GPP zur Behandlung des Asthma bronchiale bei Kindern und Jugendlichen. Monatsschrift Kinderheilk 1998; 146: $492-497$

${ }^{5}$ Bortz J, Döring N. Forschungsmethoden und Evaluation. Berlin, Heidelberg, New York: Springer, 1995

${ }^{6}$ Hindi-Alexander MC, Cropp GJA. Evaluation of a family asthma program. J Allergy Clin Immunol 1984; 74: 505 - 510

${ }^{7}$ Hughes DM, McLeod M, Garner B, Goldbloom RB. Controlled trial of a home and ambulatory program for asthmatic children. Pediatrics 1991; 87: 54-61

${ }^{8}$ The ISAAC Study Group. Lancet 1998; 351: 1225-1232

9 Juniper EF. Fragebogen zur Lebensqualität von Kindern und Jugendlichen mit Asthma. Auszufüllen durch den Interviewer. German Version. Hamilton, Ontario: McMaster-University, 1996

${ }^{10}$ Kaluza G, Schulze H-H. Evaluation von Gesundheitsförderungsprogrammen - Methodische Stolpersteine und pragmatische Empfehlungen. Zeitschrift für Gesundheitspsychologie 2000; 8: 18-24

${ }^{11}$ Lacasse Y, Wong E, Eyatt EH, King D, Cook DJ, Goldstein R. Metaanalysis of respiratory rehabilitation in chronic obstructive pulmonary disease. Lancet 1996; 348: 1115 - 1119

12 Lecheler J, Erner S. Schuldefizite chronisch asthmakranker Kinder und Jugendlicher. Monatsschr Kinderheilkunde 1991; 139: 69-72

13 Lecheler J. Sport und körperliches Training bei Kindern und Jugendlichen. In: Petro W (Hrsg.). Pneumologische Prävention und Rehabilitation. Berlin, Heidelberg, New York: Springer, 1994: 446 - 452

${ }^{14}$ Mesters I, Meertens R, Kok G, Parcel GS. Effectiveness of a multidisciplinary education protocol in children with asthma $(0-4$ years $)$ in primary health care. J Asthma 1994; 31 (5): 347-359

${ }^{15}$ Mitchell EA, Ferguson V, Norwood M. Asthma education by community child health nurses. Arch Dis Child 1986; 61: 1184-1189 
${ }^{16}$ Müller EA, Eigen J. Pediatric pulmonary function testing in asthma. Ped Clin North Am 1992; 39: 1243

${ }^{17}$ Petro W. Pneumologische Rehabilitation - was ist gesichert? Atemund Lungenkrkh 1991; 17: 43-50

18 Petermann F, Bauer CP, Kiosz D, Stachow R. Die ESTAR-Studie - Konzept und erste Ergebnisse zur Evaluation der stationären Rehabilitation bei Asthma im Kindes- und Jugendalter. Deutsche Rentenversicherung. August 2001

${ }^{19}$ Reinhardt D. Asthma bronchiale im Kindesalter. Springer, 1999

${ }^{20}$ Verband Deutscher Rentenversicherungsträger. Rahmenkonzept und indikationsspezifische Konzepte zur medizinischen Rehabilitation von Kindern und Jugendlichen in der gesetzlichen Rentenversicherung. DRV-Schriften Band 8.1998

${ }^{21}$ Sozialgesetzbuch - IX. Buch - (SGB IX - Rehabilitation und Teilhabe behinderter Menschen vom 19. Juni 2001) (BGBl I, Seite 1.045).

22 Strunck R. „National Jewish Center for Immunology and Respiratory Medicine“: Der Zugang zum schwer asthmakranken Kind. In: Petermann F, Lecheler J (Hrsg.). Asthma bronchiale im Kindes- und Jugendalter. München: Quintessenz-Verlag, 1993: 141-152

23 Strunck R, Fukuhara JT, LeBrecque JF, Mrazek DA. Outcome of longterm hospilatization for asthma in children. Journal of Allergy and Clinical Immunology 1989; 83: 17-25

${ }^{24}$ Szczepanski R. Ergebnisse einer strukturierten Asthmaschulung im Kindes- und Jugendalter. Pneumologie 1996; 50: 544-548

${ }^{25}$ Weinstein AG, McKee L, Stapleford J, Faust D. An economic evaluation of short-term inpatient rehabilitation for children with severe asthma. J Allergy Clin Immunol 1996; 98: 264-277

${ }^{26}$ Zach M. Lungenfunktionsdiagnostik im Schulkindalter In: Rieger C, v. d. Hardt H, Sennhauser FH, Wahn U, Zach M (Hrsg.). Pädiatrische Pneumologie. Berlin, Heidelberg, New York: Springer, 1999: 151 - 165

27 Zapletal A, Pragike T, Sonduk H. Lung function in children and adolescence. Methods and reference values. München, Paris, London, New York: Karger, 1979

\section{Buchbesprechung}

\section{Wissenschaft für die Westentasche \\ J. Gribbin}

Aus dem Englischen von Thorsten Schmidt

115 S., 10 Abb., Register

Originalausgabe „The Little Book of Science“, 1999

Deutsche Ausgabe: Piper Verlag GmbH, 2000 München; Kart.,

EUR 9,90 ISBN 3-492-04229-5

Gerade der „Gebildete“ läuft nur zu leicht in die „Falle des Kurzzeitdenkens", das uns heute von der Mediokratie mit Schlagworten oktroyiert wird - und so bedarf es durchaus eines „Vademecums“.

Dafür zwei Beispiele:

1. Der "Quantensprung“ - von Politikern und auch Naturwissenschaftlern - als Kriterium für eine enorme Steigerung (Verbesserung?) des Bisherigen postuliert, ist doch nur ein „Minimalbegriff“: ein "Quant“ ist die kleinstmögliche Veränderung innerhalb eines „Systems“ (Atoms), der „Quantensprung“, zudem zufallsabhängig, also nicht „groß“! Und wie steht es mit der strengen Symmetrie der drei Quark- und Leptonenpaare (gibt es vielleicht doch mehrere?), die auf eine noch tiefere „Schicht“ im Aufbau der physikalischen Welt hinweisen (von der zudem - heute - noch niemand weiß, woraus sie besteht.)?

2. Albert Einstein hat mit seiner Allgemeinen Relativitätstheorie $\left(E=m c^{2}\right)$ ein expandierendes Weltall postuliert, dessen „Inhalt" (Galaxien) sich immer weiter voneinander entferne dass somit von einem Punkt „Null“ (= Nichts?), dem „Urknall“, durch quantenphysikalische Prozesse (s.o.) das Universum entstanden sei. Als Beweise wurden das „Inflationsmodell“, Hubbles „Rotverschiebung“ und die kosmische Hintergrundstrahlung bemüht.

Auch der Autor vertritt dieses Theorem - lässt aber unbeachtet, dass dem „Big Bang“ der „Steady State“ entgegen steht und dass die „Rotverschiebung“ keineswegs gleichmäßig für alle Körper im „Raum“ gilt, in dem sich die „Materie“ zwar immer weiter voneinander entfernt, der unendliche „Raum“ selbst sich dagegen nicht auszudehnen braucht (zudem gibt zum Faktor „Zeit“ der 90. Psalm eine klare Antwort!) - und auch die physikalisch anerkannte „Chaos-Theorie“ steht dem entgegen.

Ein namhafter Physiker erklärt uns in diesem „Taschenwörterbuch" 50 mehr oder weniger bekannte Begriffe aus dem Bereich der Physik (merke: auch Chemie und damit die Biologie gehören dazu!): von der „Abstammung des Menschen“ (eines im Wald erfolglosen „Menschenaffen“) bis zum „Zweiten Hauptsatz der Thermodynamik“ (welcher Nichtphysiker versteht ihn?), von der „biologischen Zelle“ über den „kosmischen String“ bis zu den „Tachyonen“, vom „Chaos“ und dem (noch zu erforschenden) „Humangenomprojekt“ bis zu „Ypsilon-Chromosomen“.

Eine kurzweilige und doch provozierende Lektüre, die dem an den Naturwissenschaften Interessierten nicht nur Antworten gibt, sondern auch weitere Fragen aufwirft. 